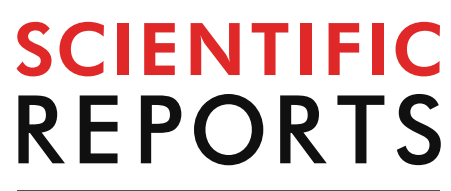

natureresearch

Check for updates

\title{
Genomic imprinting analyses
} identify maternal effects as a cause of phenotypic variability in type 1 diabetes and rheumatoid arthritis

\author{
Inga Blunk ${ }^{1}$, Hauke Thomsen ${ }^{2,3}$, Norbert Reinsch ${ }^{1}$, Manfred Mayer ${ }^{1}$, Asta Försti ${ }^{2,4,5,6}$, \\ Jan Sundquist ${ }^{4,7,8}$, Kristina Sundquist ${ }^{4,7,8}$ \& Kari Hemminki ${ }^{2,4,9}$
}

Imprinted genes, giving rise to parent-of-origin effects (POEs), have been hypothesised to affect type 1 diabetes (T1D) and rheumatoid arthritis (RA). However, maternal effects may also play a role. By using a mixed model that is able to simultaneously consider all kinds of POEs, the importance of POEs for the development of T1D and RA was investigated in a variance components analysis. The analysis was based on Swedish population-scale pedigree data. With $P=0.18$ (T1D) and $P=0.26$ (RA) imprinting variances were not significant. Explaining up to $19.00 \%( \pm 2.00 \%)$ and $15.00 \%( \pm 6.00 \%)$ of the phenotypic variance, the maternal environmental variance was significant for T1D $\left(P=1.60 \times 10^{-24}\right)$ and for RA $(P=0.02)$. For the first time, the existence of maternal genetic effects on RA was indicated, contributing up to $16.00 \%( \pm 3.00 \%)$ of the total variance. Environmental factors such as the social economic index, the number of offspring, birth year as well as their interactions with sex showed large effects.

The failure of the immune system to distinguish self from non-self antigens is the basis for autoimmune disorders (AIs) ${ }^{1}$. Type I diabetes (T1D) is an AI that causes chronic destruction of pancreatic islet $B$-cells and hyperglycemia due to reduced insulin production ${ }^{2}$. With the incidence said to be increasing by 3-4\% yearly, more than 20 million individuals are estimated to have T1D worldwide ${ }^{3}$. Rheumatoid arthritis (RA) is associated with autoantigen presentation with antigen specific $\mathrm{T}$ and $\mathrm{B}$ cell activation and aberrant inflammatory cytokine production. Consequences thereof include synovitis, proliferation of synovia and cartilage, and subchondral bone destruction ${ }^{4}$. The occurrence of RA is relatively constant and ranges between 0.5 and $1.0 \%$ in European and North-American populations ${ }^{5}$. The exact etiology of T1D and RA remains largely unknown ${ }^{1}$, however, a complex interplay of genetic, environmental, and epigenetic factors is assumed ${ }^{4,6,7}$.

With regard to genetic factors, the strongest effects have been found within the major histocompatibility complex or human leukocyte antigen system. T1D and RA show genetic overlap in terms of associations within HLA, PTPN22, CTLA4, and TAGAP ${ }^{8}$. Causal loci explain over $80 \%$ of T1D heritability which reportedly ranges between 40 and $92 \%{ }^{3}$. For RA, associated variants outside and inside of the major histocompatibility complex region explain about $5 \%$ and $60 \%$ of the heritability ${ }^{9,10}$. Heritability estimates range between 12 and $60 \%{ }^{11-13}$.

As disorder concordance rates in monozygotic twins have been observed to be less than $100 \%$, AIs are assumed to be subject to epigenetic modifications ${ }^{4,14-16}$. Perhaps the best-known example for all epigenetic phenomena is imprinting, in which the expression of genes is either maternally or paternally inactivated. Inactivation

\footnotetext{
${ }^{1}$ Institute of Genetics and Biometry, Leibniz Institute for Farm Animal Biology (FBN), Wilhelm-Stahl-Allee 2, 18196 Dummerstorf, Germany. ${ }^{2}$ Division of Molecular Genetic Epidemiology, German Cancer Research Centre (DKFZ), Heidelberg, Germany. ${ }^{3}$ GeneWerk GmbH, Heidelberg, Germany. ${ }^{4}$ Center for Primary Health Care Research, Lund University, Malmö, Sweden. ${ }^{5}$ Hopp Children's Cancer Center (KiTZ), Heidelberg, Germany. ${ }^{6}$ Division of Pediatric Neurooncology, German Cancer Research Center (DKFZ), German Cancer Consortium (DKTK), Heidelberg, Germany. ${ }^{7}$ Department of Family Medicine and Community Health, Department of Population Health Science and Policy, Icahn School of Medicine at Mount Sinai, New York, USA. ${ }^{8}$ Center for Community-Based Healthcare Research and Education (CoHRE), Department of Functional Pathology, School of Medicine, Shimane University, Izumo, Japan. ${ }^{9}$ Faculty of Medicine and Biomedical Center in Pilsen, Charles University in Prague, Pilsen, Czech Republic. ${ }^{\varpi}$ email: blunk@fbn-dummerstorf.de
} 
can either be full or partial ${ }^{17}$. Partial imprinting occurs when the inactivation of alleles is not complete. For example, loci may be imprinted in a tissue-specific manner ${ }^{18}$, or the imprinting status varies over time during the developmental stages ${ }^{19}$. As they appear as phenotypic differences between heterozygotes depending on their parental allele origin, imprinting effects belong to the class of parent-of-origin effects (POEs) ${ }^{20}$. Imprinting has been identified in mammals, insects, and plants ${ }^{21}$. It is nevertheless assumed that less than $1 \%$ of all genes in mammals are imprinted ${ }^{20,22}$, however, they have important functions in stem cells, neuronal differentiation, development, and growth ${ }^{22,23}$. In humans, imprinted genes are associated with diseases such as Prader-Willi syndrome ${ }^{24}$, Angelman syndrome ${ }^{25}$, and cancer ${ }^{26,27}$. They are also assumed to affect susceptibility to diabetes. This assumption originates from observations that T1D is preferentially expressed by children of T1Daffected fathers ${ }^{28-30}$. Whether this observation is due to imprinting or other factors is not clear since findings are contradictory ${ }^{29,31}$. With regard to RA, the existence of imprinting has been discussed since its incidence is considerably higher in women than in men $^{32}$. However, imprinting studies are rare and results have been inconclusive; the role of imprinting in RA susceptibility is therefore not yet understood ${ }^{33,34}$. Imprinted genes are difficult to detect in conventional association studies as their effects depend on the parental origin of the risk allele ${ }^{35}$. The incorporation of knowledge on whether imprinting affects susceptibility to T1D and RA could increase the power to find causal genes ${ }^{34}$. Moreover, the development of therapeutic approaches targeting these genes or their regulators could be improved ${ }^{33}$. Therefore, the first goal of this study was to investigate the impact of imprinting on the susceptibility to T1D and RA in a variance components analysis by applying a unique mixed model (imprinting model). The model allows for the simultaneous consideration of all kinds of imprinting patterns (full, partial, maternal, and paternal). As it has never been applied to human population data before, it opens up new opportunities for understanding the etiology of T1D and RA ${ }^{17,36-40}$.

In an imprinting variance components analysis, maternal effects must be accounted for in the model to avoid inflated estimates ${ }^{41}$. The second research goal was therefore to incorporate maternal effects into the statistical model; not only to prevent biases in the imprinting variances, but also to investigate the maternal contribution to T1D and RA susceptibility. Like imprinting effects, maternal effects contribute to the broader class of POEs. However, their variation is assigned to the environmental contribution to the phenotypic variance. According to Falconer ${ }^{42}$, they are defined as prenatal and postnatal effects on offspring and can have two main components. The first component is the maternal genotypic effect on, for example, the birthweight of her children (maternal genetic effect $)^{43}$. The second component is the maternal environmental effect on the birthweight of her offspring ${ }^{42}$. This component refers to the permanent environmental effects of the mother on all of her offspring and can therefore also be considered a shared household effect ${ }^{42}$. Although T1D and RA differ in their average age of onset, attention must be given to the maternal contribution in the development of both diseases since early environmental factors can permanently modify the development of the immune system ${ }^{44}$. The imprinting model is able to separate maternal effects from maternal imprinting effects, allowing the first imprinting variance components analysis to be performed in human population genetics.

The third goal of this study was to gain knowledge on the importance of sex and environmental triggers such as birth year, social economic index, and the number of offspring on the susceptibility to T1D and RA. Overall, this study brought to light the complex interplay between genetic, epigenetic and environmental factors in the development of autoimmunity.

\section{Theory}

A unique mixed model, previously used on animal data, was applied to investigate the existence of imprinting ${ }^{36-40,45}$. The advantage this model confers is that it is able to simultaneously consider all kinds of imprinting (i.e. maternal, paternal, full, and partial imprinting) in its analyse ${ }^{17}$, ultimately separating maternal imprinting effects from maternal "non-imprinting" effects (e.g., maternal environmental and maternal genetic effects). This was not possible with previous population-scale imprinting analyses models, for example, that of Engellandt and Tier ${ }^{46}$. Our imprinting model estimates two parental gametic variances and one covariance simultaneously. It is written as:

$$
\boldsymbol{Y}=\boldsymbol{X} \boldsymbol{b}+\boldsymbol{Z}_{\boldsymbol{s}} \boldsymbol{g}_{\boldsymbol{s}}+\boldsymbol{Z}_{\boldsymbol{d}} \boldsymbol{g}_{\boldsymbol{d}}+\boldsymbol{e}, \quad \text { (imprinting model) }
$$

where $\boldsymbol{Y}$ is a vector of the response variable; $\boldsymbol{b}$ is a vector of fixed effects; $\boldsymbol{g}_{\boldsymbol{s}}$ is the vector of random gametic effects under a paternal expression pattern; $\boldsymbol{g}_{\boldsymbol{d}}$ is the vector of random gametic effects under a maternal expression pattern; $\boldsymbol{X}, \boldsymbol{Z}_{\boldsymbol{s}}$, and $\boldsymbol{Z}_{\boldsymbol{d}}$ are the corresponding incidence matrices; and $\boldsymbol{e}$ is the vector of random residuals. The variance-covariance structure is:

$$
\operatorname{Var}\left[\begin{array}{l}
\boldsymbol{g}_{s} \\
\boldsymbol{g}_{\boldsymbol{d}}
\end{array}\right]=G \otimes\left[\begin{array}{cc}
\sigma_{s}^{2} & \sigma_{s d} \\
\sigma_{s d} & \sigma_{d}^{2}
\end{array}\right],
$$

where $\sigma_{s}^{2}$ and $\sigma_{d}^{2}$ are the gametic variances and $\sigma_{s d}$ is the covariance. Matrix $\boldsymbol{G}$ is the gametic relationship matrix reflecting the relationships between the gametes of all individuals in a pedigree. It is therefore twice the size of the number of individuals included in the analysis ${ }^{47,48}$. The symbol $\otimes$ denotes the Kronecker product. The imprinting effect is defined as the vector of differences $\left(\boldsymbol{g}_{\boldsymbol{s}}-\boldsymbol{g}_{\boldsymbol{d}}\right)$ and the corresponding variance of differences is $\sigma_{i}^{2}=\sigma_{s}^{2}+\sigma_{d}^{2}-2 \sigma_{s d}$, which represents the imprinting variance. Where no imprinting is observed, $\sigma_{s}^{2}=\sigma_{d}^{2}=\sigma_{s d}$ and $\sigma_{i}^{2}=0$. 
(a)

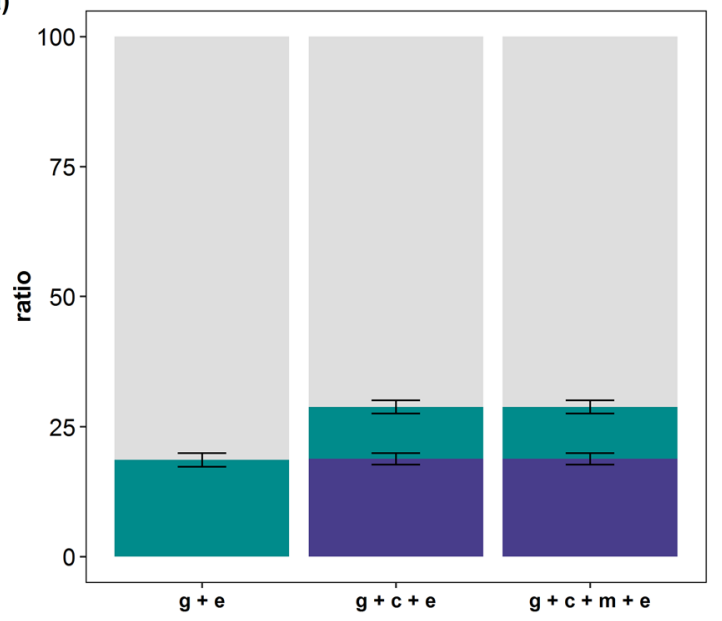

(b)

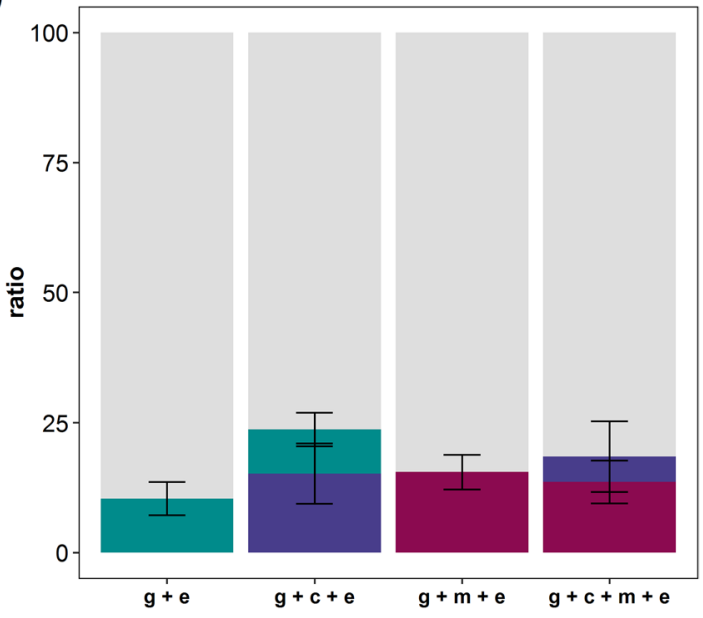

Figure 1. Phenotypic variance of type 1 diabetes (a) and rheumatoid arthritis (b) is partitioned into the residual variance (gray), additive genetic variance (green), maternal environmental variance (blue), and maternal genetic variance (red). The variance components were estimated using models with a gametic effect $(g)$, a maternal environmental effect $(c)$, a maternal genetic effect $(m)$, and a residual $(e)$. Standard errors are indicated by error bars.

\section{Results}

Parent-of-origin effects. Type 1 diabetes. Genomic imprinting. Using a REML log-likelihood ratio test (RLRT), the significance of the imprinting variance was tested by comparing the logarithmic value of the restricted maximum likelihood (REML log-likelihood) of the linear imprinting model to the REML log-likelihood outcome of a corresponding linear Mendelian model (equivalent null model that assumes the non-existence of imprinting). At a 5\% significance level, the analysis revealed that imprinted genes did not significantly contribute to the total genetic variance in T1D susceptibility in the Swedish population data $(P=0.18)$.

Maternal effects. Initially, data were analysed using linear models in order to test the significance of the variance components. First, a linear model that ignored maternal effects was applied, i.e. only the genetic effect of the individual was included in the model (Mendelian model 1). This led to a T1D heritability estimate $\left(h^{2}\right)$ of 0.19 $\left( \pm 0.1 \times 10^{-1}\right)$, i.e. $19 \%$ of the phenotypic variation in T1D is due to the variation in genetic factors in the analysed population (Fig. 1). Adding a maternal environmental effect to the model (Mendelian model 2) revealed significant maternal environmental variance with $P=1.60 \times 10^{-24}$. The relative maternal T1D environmental variance was $0.19\left( \pm 0.2 \times 10^{-1}\right)$, i.e. $19 \%$ of the phenotypic variance in T1D is due to the variation in maternal environmental effects (Fig. 1). Heritability was reduced to $0.10\left( \pm 0.1 \times 10^{-1}\right)$. Augmentation of Mendelian model 2 by the maternal genetic effect (Mendelian model 3) did not change the REML log-likelihood or variance component ratios (Fig. 1). More detailed information on the variance component estimates in T1D and REML log-likelihood models is provided in Supplementary Table S1.

In addition to the linear models, threshold models were applied to account for the binary nature of the phenotypic traits. However, each of the threshold models could only pick up one variance component, i.e. with the addition of parameters, the same amount of variation was explained by additive genetic effects, then by maternal environmental effects, and then by maternal genetic effects (Table 1).

Rheumatoid arthritis. Genomic imprinting. As maternally derived environmental and genetic effects could not be unambiguously disentangled, the imprinting model was applied in two forms: (a) with only the maternal environmental effect in addition to the two parental gametic effects, and (b) with only the maternal genetic effect in addition to the two parental gametic effects. The RLRT of model version (a) did not indicate significant imprinting variance $(P=0.26)$. Model version (b) led to a REML log-likelihood of $8,408.70$, which was slightly smaller than the REML log-likelihood obtained from the corresponding null model containing a gametic effect and a maternal genetic effect $(8,408.88)$. Because the addition of a parameter to a model should result in an REML log-likelihood value either being equal to or larger than that found here, these results could indicate a flat likelihood surface or numerical inaccuracies.

Maternal effects. Maternal effects were initially ignored (Mendelian model 1), which resulted in an $h^{2}$ value of $0.10\left( \pm 0.3 \times 10^{-1}\right.$; Fig. 1). Following the inclusion of a maternal environmental effect (Mendelian model 2), the $h^{2}$ value was reduced to $0.85 \times 10^{-1}\left( \pm 0.3 \times 10^{-1}\right)$. The corresponding maternal variance component was significant at a $5 \%$ significance level $(P=0.02)$. The relative maternal environmental variance was $0.15\left( \pm 0.6 \times 10^{-1}\right.$; Fig. 1). While the REML log-likelihood value was not significantly altered upon addition of the maternal genetic effect $(P=0.21$; Mendelian model 3$)$, the relative maternal RA genetic variance estimate was $0.14\left( \pm 0.4 \times 10^{-1}\right.$; Fig. 1), the $h^{2}$ estimate dropped to zero and the relative maternal environmental variance was reduced from 0.15 $\left( \pm 0.6 \times 10^{-1}\right)$ to $0.49 \times 10^{-1}\left( \pm 0.7 \times 10^{-1}\right)$. To investigate the importance of maternal genetic effects in more detail, 


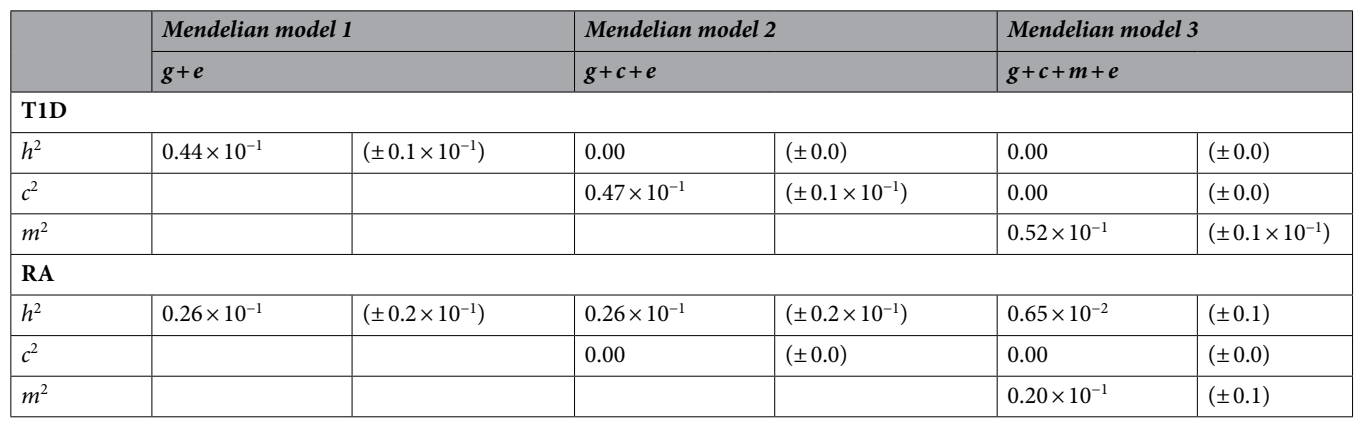

Table 1. Heritability $\left(h^{2}\right)$, relative maternal environmental variance $\left(c^{2}\right)$, and relative maternal genetic variance $\left(m^{2}\right)$ for type 1 diabetes (T1D) and rheumatoid arthritis (RA) estimated using threshold models with a gametic effect $(g)$, a maternal environmental effect $(c)$, a maternal genetic effect $(m)$, and a residual effect $(e)$. Standard errors are in parentheses.

a linear model that corresponded to the Mendelian model 2 but substituted the maternal environmental effect with maternal genetic effect was applied. The application of this model resulted in a relative maternal genetic variance of $0.16\left( \pm 0.3 \times 10^{-1}\right)$ and an $h^{2}$ estimate of zero (Fig. 1). The RLRT indicated a significantly better fit in comparison to Mendelian model $1(P=0.01)$. Comparing the results to those associated with Mendelian model 3, the REML log-likelihood was not significantly different $(P=0.53)$. Detailed information on RA variance component estimates and REML log-likelihoods of the models is provided in Supplementary Table S2.

The threshold version of Mendelian model 1 resulted in an $h^{2}$ of $0.26 \times 10^{-1}\left( \pm 0.2 \times 10^{-1}\right)$. As an equal additive genetic variance, and thus the same $h^{2}$, was found using the threshold version of Mendelian model 2, maternally derived environmental factors appeared not to play a role (Table 1) in RA. However, when the maternal genetic effect was added (Mendelian model 3) the $h^{2}$ value was $0.65 \times 10^{-2}( \pm 0.1)$, while the maternal environmental variance remained zero and the relative maternal genetic variance was $0.20 \times 10^{-1}( \pm 0.1$; Table 1$)$.

Environmental and sex effects. Type 1 diabetes. Birth year. Across all models (including linear and threshold models), the effects of the year of birth (ranging from 1944 to 2012) were shown to differ significantly $\left(P<1.00 \times 10^{-3}\right.$; Table 2; Supplementary Table S3). Effects increased until the end of the 1950s and started declining slightly at the beginning of the 1960s. The effects increased after 1972 until the mid-1980s, declined again until the mid-1990s, and have been increasing ever since (Fig. 2). With the exception of a strong increase of effects and standard errors in 1992 when applying the threshold models (data not shown), trends observed and effects generated under the threshold models were in accordance with those observed for the linear models.

Social economic index of the mother. When considering the social economic index (SEI), analyses revealed that the effects of the mother's SEI differed significantly for T1D with $P$ values ranging from $2.56 \times 10^{-12}$ to $1.13 \times 10^{-9}$ across all models (Table 2; Supplementary Table S3). Although small, the largest effect $\left(0.02 ; \pm 3.00 \times 10^{-3}\right)$ was found for the intermediate group of non-manual employees (code 4$)$. The lowest effect $\left(-2.00 \times 10^{-3} ; \pm 5.00 \times 10^{-3}\right)$ was found for professionals as well as higher civil servants and executives (code 5).

Medical region. To investigate the effect of geographical location on T1D susceptibility, medical regions were used. Using linear and threshold models, effects differed significantly for T1D across medical regions with $P$ values ranging from $2.12 \times 10^{-156}$ to $4.60 \times 10^{-104}$ (Table 2; Supplementary Table S3). As depicted in Fig. 3, effect sizes varied widely across Sweden.

Sex. A slight male skew towards T1D was observed (14,626 male vs. 12,629 female), with significantly different effects seen across all models for sex. $P$ values ranged from $8.05 \times 10^{-225}$ to $3.23 \times 10^{-20}$ (Table 2; Supplementary Table S3). The analyses further revealed significant interactions between sex and birth year with $P$ values ranging from $1.50 \times 10^{-50}$ to $3.45 \times 10^{-11}$ (Table 2; Supplementary Table S3). Minimal changes were found for estimates across models. The effect of male sex on T1D increased proportionally with birth year starting in 1965, reaching its highest point in the late 1970s, and declined until no interactions could be observed in 1990 (Supplementary Fig. S1).

Rheumatoid arthritis. Birth year. Ranging from 1939 to 2007, the effects of birth year differed significantly across all models with $P$ values ranging from $3.34 \times 10^{-265}$ to $2.64 \times 10^{-24}$ (Table 2; Supplementary Table S3). Negative effects were observed from 1939 with the lowest point obtained in 1958. Since then, RA susceptibility has increased with a positive effect being observed in 1963; a trend that continued until the end of the 1970s. The trend remained constant for approximately 10 years, with a slight increase noticeable towards the end of the 1980s. Increasing standard errors must however be noted (Fig. 2). While a similar trend was observed for the threshold models (data not shown), effects increased in 1974 and remained constant before decreasing in 1991. Large standard errors were observed for effects after 1973. 


\begin{tabular}{|c|c|c|c|c|c|c|c|c|c|c|c|c|c|}
\hline \multirow[b]{3}{*}{ Effect } & \multirow[b]{3}{*}{$D F$} & \multicolumn{3}{|c|}{ Mendelian model 1} & \multicolumn{3}{|c|}{ Mendelian model 2} & \multicolumn{3}{|c|}{ Mendelian model 3} & \multicolumn{3}{|c|}{ imprinting model } \\
\hline & & \multicolumn{3}{|l|}{$g+e$} & \multicolumn{3}{|l|}{$g+c+e$} & \multicolumn{3}{|c|}{$g+c+m+e$} & \multicolumn{3}{|c|}{$g_{s}+g_{d}+c+e$} \\
\hline & & $D F_{d e n}$ & $F$ & $P$ & $D F_{d e n}$ & $F$ & $P$ & $D F_{d e n}$ & $F$ & $P$ & $D F_{d e n}$ & $F$ & $P$ \\
\hline \multicolumn{14}{|l|}{ T1D } \\
\hline Birth year & 67 & $68,461.1$ & $1,696.76$ & 0.00 & $69,638.8$ & $1,579.83$ & 0.00 & $69,631.0$ & $1,579.70$ & 0.00 & $69,586.8$ & $1,577.98$ & 0.00 \\
\hline $\mathrm{SEI}_{\text {mother }}$ & 5 & $70,870.5$ & 12.35 & $5.35 \times 10^{-12}$ & $68,490.8$ & 12.64 & $2.68 \times 10^{-12}$ & $68,489.6$ & 12.64 & $2.68 \times 10^{-12}$ & $68,395.0$ & 12.66 & $2.56 \times 10^{-12}$ \\
\hline Sex & 1 & $70,917.7$ & $1,032.05$ & $8.05 \times 10^{-225}$ & $70,779.3$ & 971.73 & $6.88 \times 10^{-212}$ & $70,779.3$ & 971.68 & $7.05 \times 10^{-212}$ & $70,464.8$ & 892.65 & $6.45 \times 10^{-195}$ \\
\hline Sexbirth year & 67 & $69,252.5$ & 6.12 & $1.50 \times 10^{-50}$ & $70,163.3$ & 6.12 & $1.50 \times 10^{-50}$ & $70,157.3$ & 6.12 & $1.50 \times 10^{-50}$ & $70,105.4$ & 6.11 & $1.98 \times 10^{-50}$ \\
\hline Medical region & 25 & $50,691.5$ & 32.97 & $2.12 \times 10^{-156}$ & $47,088.9$ & 32.50 & $7.34 \times 10^{-154}$ & $47,120.5$ & 32.50 & $7.33 \times 10^{-154}$ & $47,038.1$ & 32.52 & $5.80 \times 10^{-154}$ \\
\hline Years $_{\text {obs }}$ & 3 & $70,670.0$ & $1,086.26$ & 0.00 & $70,689.1$ & $1,086.68$ & 0.00 & $70,687.3$ & $1,086.68$ & 0.00 & $70,674.5$ & $1,086.92$ & 0.00 \\
\hline \multicolumn{14}{|l|}{ RA } \\
\hline Birth year & 56 & $20,417.2$ & 26.69 & $3.34 \times 10^{-265}$ & $20,513.0$ & 25.01 & $7.06 \times 10^{-247}$ & $20,356.1$ & 24.54 & $1.14 \times 10^{-241}$ & $20,067.2$ & 23.07 & $1.84 \times 10^{-225}$ \\
\hline SEI & 5 & $20,858.0$ & 9.89 & $1.84 \times 10^{-9}$ & $20,853.7$ & 9.93 & $1.68 \times 10^{-9}$ & $20,851.0$ & 9.94 & $1.64 \times 10^{-9}$ & $20,811.1$ & 9.98 & $1.49 \times 10^{-9}$ \\
\hline $\mathrm{SEI}_{\text {mother }}$ & 5 & $20,845.5$ & 2.98 & $0.11 \times 10^{-1}$ & $20,194.7$ & 2.99 & $0.11 \times 10^{-1}$ & $20,068.6$ & 2.95 & $0.12 \times 10^{-1}$ & $20,134.7$ & 2.98 & $0.11 \times 10^{-1}$ \\
\hline Sex & 1 & $20,852.5$ & 686.42 & $6.86 \times 10^{-149}$ & \begin{tabular}{|l|}
$20,829.9$ \\
\end{tabular} & 680.46 & $1.24 \times 10^{-147}$ & \begin{tabular}{|l|}
$20,856.2$ \\
\end{tabular} & 642.81 & $1.06 \times 10^{-139}$ & $20,850.8$ & 620.47 & $5.54 \times 10^{-135}$ \\
\hline Sexbirth year & 56 & $20,516.0$ & 1.38 & $0.32 \times 10^{-1}$ & $20,607.1$ & 1.38 & $0.32 \times 10^{-1}$ & $20,526.9$ & 1.38 & $0.32 \times 10^{-1}$ & $20,170.0$ & 1.39 & $0.29 \times 10^{-1}$ \\
\hline Sex ${ }^{\star}$ SEI & 5 & $20,833.9$ & 6.29 & $7.71 \times 10^{-6}$ & $20,798.3$ & 6.24 & $8.64 \times 10^{-6}$ & $20,815.4$ & 6.26 & $8.25 \times 10^{-6}$ & $20,729.7$ & 6.26 & $8.25 \times 10^{-6}$ \\
\hline Sexno. offspring & 11 & $20,799.0$ & 8.58 & $2.51 \times 10^{-15}$ & $20,605.9$ & 8.59 & $2.39 \times 10^{-15}$ & $20,587.5$ & 8.57 & $2.64 \times 10^{-15}$ & $20,256.6$ & 8.58 & $2.52 \times 10^{-15}$ \\
\hline No. offspring & 11 & $20,681.9$ & 175.65 & 0.00 & $20,472.8$ & 175.61 & 0.00 & $20,537.8$ & 175.55 & 0.00 & $20,478.7$ & 175.70 & 0.00 \\
\hline Medical region & 25 & $16,126.9$ & 1.81 & $0.80 \times 10^{-2}$ & $16,090.3$ & 1.81 & $0.80 \times 10^{-2}$ & $17,638.6$ & 1.82 & $0.70 \times 10^{-2}$ & $15,772.9$ & 1.84 & $0.65 \times 10^{-2}$ \\
\hline Single child & 1 & $20,857.7$ & 6.33 & $0.12 \times 10^{-1}$ & $20,804.6$ & 6.14 & $0.13 \times 10^{-1}$ & $20,537.8$ & 6.09 & $0.14 \times 10^{-1}$ & $20,760.6$ & 6.14 & $0.13 \times 10^{-1}$ \\
\hline Years $_{\text {obs }}$ & 3 & $20,653.1$ & 13.66 & $6.73 \times 10^{-9}$ & $20,688.3$ & 13.76 & $5.82 \times 10^{-9}$ & $20,520.3$ & 13.68 & $6.54 \times 10^{-9}$ & $20,314.2$ & 13.60 & $7.35 \times 10^{-9}$ \\
\hline
\end{tabular}

Table 2. Overview of incremental Wald $F$ values $(F)$, number of numerator degrees of freedom $(D F)$, number of denominator degrees of freedom $\left(D F_{d e n}\right)$, and the $P$ values $(P)$ for all fixed effects on type 1 diabetes (T1D) and rheumatoid arthritis (RA), which were sex, birth year, social economic index (SEI), number of offspring (no. offspring), medical region, $\mathrm{SEI}$ of the mother $\left(\mathrm{SEI}_{\text {mother }}\right.$ ), years under observation ( years $\left._{\mathrm{obs}}\right)$, and whether an individual was a single child or not (single child). Linear mixed models were used containing a gametic effect $(g)$, a gametic effect as father $\left(g_{s}\right)$, a gametic effect as mother $\left(g_{d}\right)$, a maternal environmental effect $(c)$, a maternal genetic effect $(m)$, and a residual effect $(e)$.

SEI of individual. The SEIs of individuals significantly differed for RA with an average $P$ value of $1.66 \times 10^{-9}$ for linear models and $P=0.01$ for threshold models (Table 2; Supplementary Table S3). Effect estimates varied little across models. The largest effect $(0.11 ; \pm 0.03)$ was found for unskilled or semi-skilled workers, while the lowest effect $\left(0.02 ; \pm 8.00 \times 10^{-3}\right)$ was observed for foremen in industrial production and assistant non-manual employees.

SEI of the mother. Maternal SEIs had a small but significant effect on RA susceptibility in offspring under both the linear and threshold models. $P$ values ranged from 0.01 to 0.02 (Table 2; Supplementary Table S3). Effects varied little across models with similar estimates being calculated. The lowest effect was found for foremen in industrial production and assistant non-manual employees $(-0.03 ; \pm 0.01)$, followed by skilled manual workers $(-0.01 ; \pm 0.01)$. Except for the unknown SEI group, the highest effect was found for the group of professionals as well as higher civil servants and executives $\left(4.00 \times 10^{-3} ; \pm 0.01\right)$.

Number of offspring. In the dataset, women had an average number of 1.94 children (ranging from zero to 11 children; $s d=1.25$ ), while men had an average number of 2.04 children (ranging from zero to 11 children; $s d=1.37)$. The number of offspring affected RA development significantly across all models $\left(P<1.00 \times 10^{-3}\right)$. An inverse and almost linear relationship between RA susceptibility and the number of children is depicted in Fig. 4. While effects greater than zero were estimated for individuals with zero, one or two children, decreasing effects were observed below zero for individuals with more than two children.

Medical region. Medical regions, serving as the proxy for residential and geographic location, differed significantly in their impact on RA with $P$ values ranging from $0.70 \times 10^{-2}$ to 0.01 across all models (Table 2; Supplementary Table S3). Effect sizes varied widely across Sweden and were generally small with large standard errors (Supplementary Fig. S2).

Single child. We found that being a single child or having siblings made a significant difference regarding RA susceptibility with $P$ values ranging from 0.01 to 0.02 across the models (Table 2; Supplementary Table S3). For the linear models, the mean estimated effect of being a single child was $-0.02\left( \pm 9.00 \times 10^{-3}\right)$, while an effect of $-0.15( \pm 0.06)$ was observed under the threshold models. 

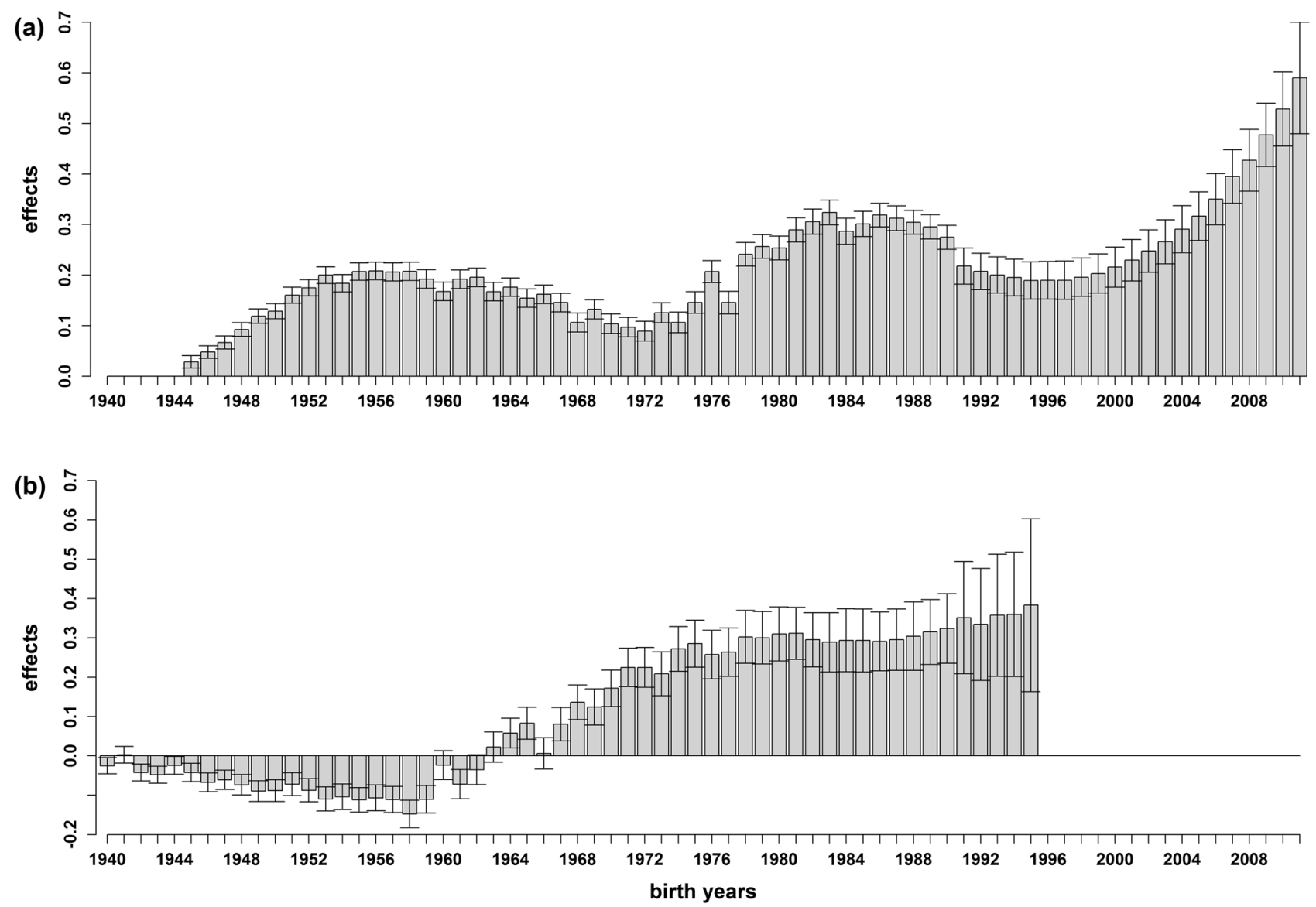

Figure 2. Effects of birth years on the susceptibility to type 1 diabetes (a) and rheumatoid arthritis (b). For type 1 diabetes, effects were estimated using a linear mixed model that includes a random gametic effect and a random maternal environmental effect. For rheumatoid arthritis a random maternal genetic effect was added. The standard errors are indicated by error bars.

Sex. The incidence of RA was considerably higher in women than in men with 11,442 female and 4,408 male cases, respectively. Sex effects were significantly different with $P$ values ranging from $6.86 \times 10^{-149}$ to $6.61 \times 10^{-101}$ across models (Table 2; Supplementary Table S3). Interactions between sex and birth year were significant only under the linear models $(P=0.03 ; P=1.00$ in the threshold models). No clear trend was visible for interaction effects amongst male patients (Supplementary Fig. S1). Significant interactions between sex and SEI (average $P$ value of $4.56 \times 10^{-6}$ ) as well as between sex and the number of offspring were observed (average $P$ value of $2.55 \times 10^{-15}$ ). The latter interaction was not significant using threshold models (average $P$ value of 0.48 ; Supplementary Table S3).

Linear versus threshold model. Firstly, the concordance across the linear and threshold model results were investigated by comparing the predicted genetic values under Mendelian model 1 . High Pearson correlation coefficients were obtained with $r=0.99$ for both T1D and RA. The linear relationships are shown in Supplementary Fig. S3. Secondly, threshold genetic values were fitted using the linear genetic values as independent variables. These results and their respective residual values are shown in Supplementary Fig. S3. The residual variation was fairly constant with some outliers observed over the entire range for T1D and RA.

\section{Discussion}

Parent-of-origin effects. Our finding that imprinting did not seem to affect T1D susceptibility supported previous findings by McCarthy et al. (1991), who analysed the importance of imprinting in an epidemiological study of clinical data from the Children's Hospital of Pittsburgh IDDM Registry in Pennsylvania, USA ${ }^{29}$. They rejected the imprinting hypothesis and suggested that other genetic and environmental factors may have caused disease occurrence ${ }^{29}$. In addition, Guo and Tuomilehto (2002) stated that the male preponderance in T1D prevalence, fecundity differences, misclassification of T1D and birth order effects could have led to a higher T1D-prevalence in children of T1D-affected fathers ${ }^{30}$. In contrast, a genome-wide association study of European T1D patients showed that an imprinted T1D-associated locus was located within the maternally expressed MEG3 gene ${ }^{31}$.

With regard to RA our findings are consistent with observations made by Zhou et al. (2007), who found that imprinting is unlikely to affect the susceptibility to $\mathrm{RA}^{34}$. In most tissues, the IGF2 (insulin-like growth factor 


\section{Medical regions}

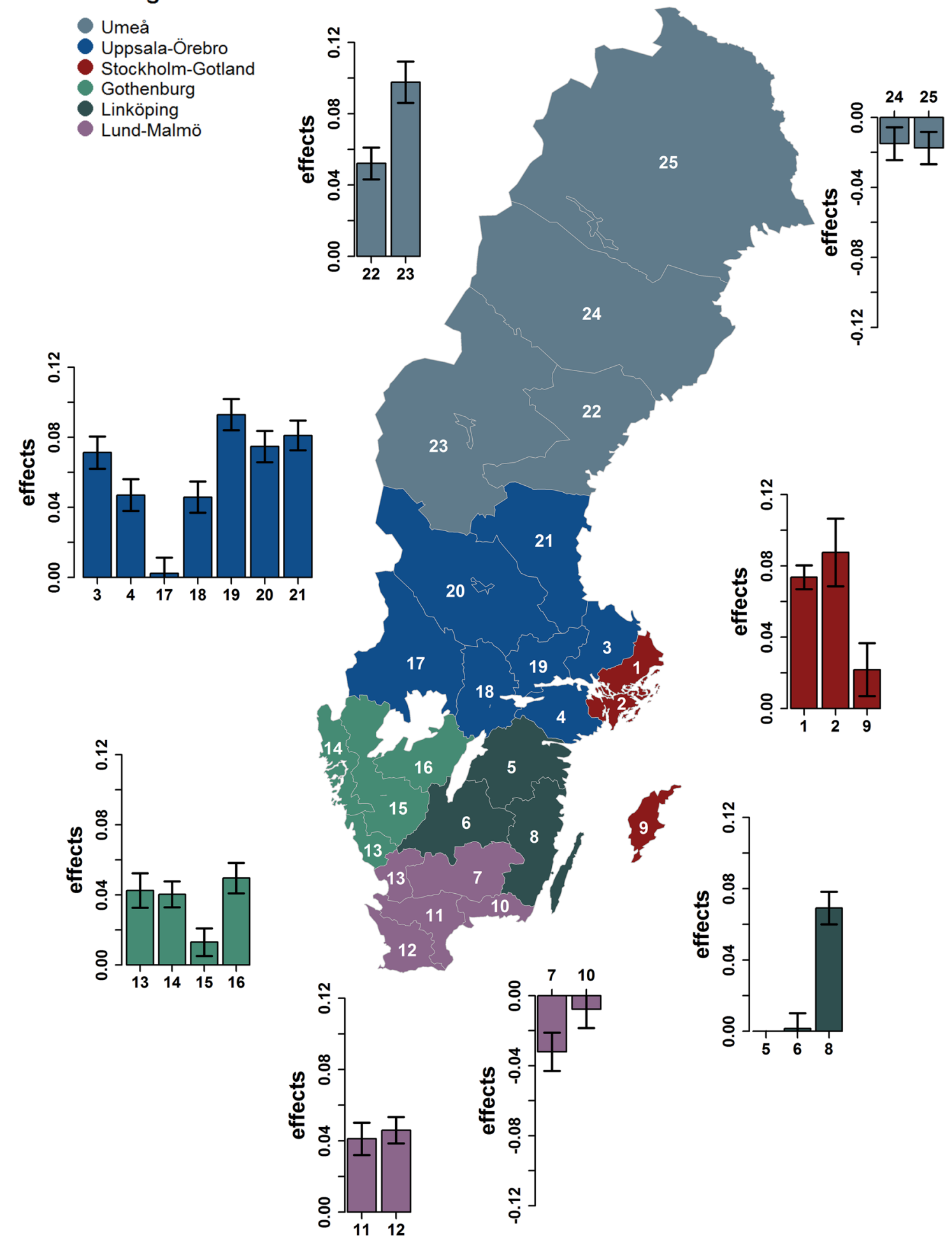

Figure 3. Effects of counties on the susceptibility to type 1 diabetes summarised into medical regions in Sweden. Effects were estimated using a linear mixed model that includes a random gametic effect and a random maternal environmental effect. Standard errors are indicated by error bars. Coordinates of Sweden were downloaded from https://www.scb.se/hitta-statistik/regional-statistik-och-kartor/regionala-indelningar/digit ala-granser/ (accessed in November 2019) in the ArcView-shape format. Data were edited using the "readOGR" function implemented in the R-package "rgdal" version 1.4-8 (Bivand, R., Keitt, T. \& Rowlingson, B. rgdal: Bindings for the 'Geospatial' Data Abstraction Library. R package version 1.4-8. (2019)) which was used in R version 3.6.1 (R Core Team (2019). R: A Language and Environment for Statistical Computing (R Foundation for Statistical Computing, Vienna, Austria)). 


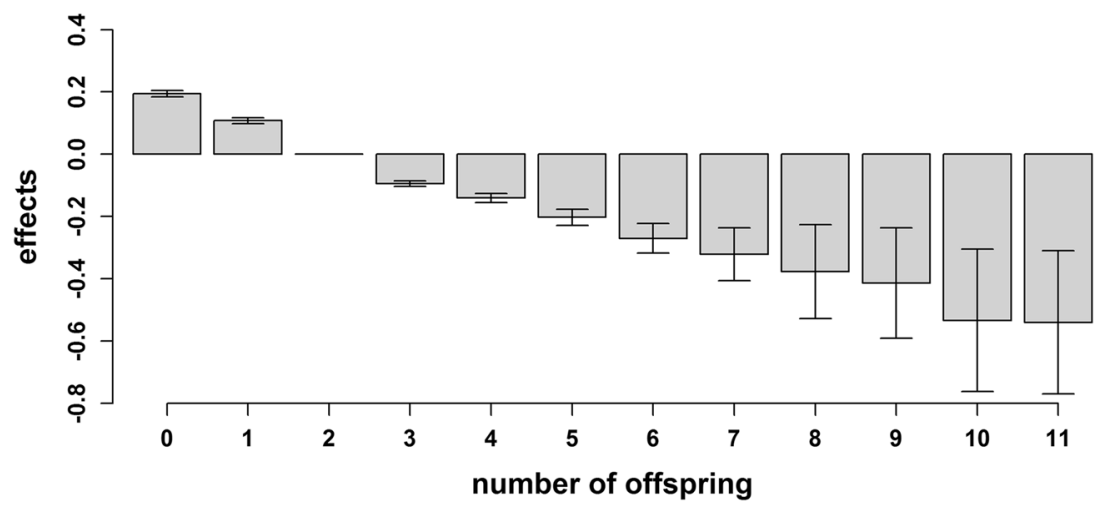

Figure 4. Effects of the number of offspring on the susceptibility to rheumatoid arthritis estimated using a linear mixed model that includes a random gametic, a random maternal environmental, and a random maternal genetic effect. The standard errors are indicated by error bars.

2) gene is only paternally expressed, i.e. the maternal allele is imprinted. Martin-Trujillo et al. (2010) found an increased expression of IGF2 in a subset of RA fibroblast-like synoviocytes ${ }^{33}$. This cell type forms the synovial intimal lining and contributes to cartilage destruction and synovial inflammation ${ }^{49}$. The authors reported that IGF2-linked "loss of imprinting" was responsible for the increased expression that contributed to the autonomous growth of RA fibroblast-like synoviocytes ${ }^{33}$. These findings demonstrated the effects of partially imprinted loci on RA susceptibility.

In our study, the hypothesis that imprinted loci were the major cause influencing T1D and RA susceptibility was rejected. However, where a small number of fully or partially imprinted genes with small or moderate effect sizes does exist, the relative imprinting variance (ratio between the imprinting variance and the total additive genetic variance) is expected to be small. Although the imprinting model considers all kinds of imprinting ${ }^{17,36,37}$, our study may have been underpowered and therefore unable to obtain statistical significance. Power to detect significant imprinting variances depends on both the $h^{2}$ value of a trait and relative imprinting variance. In this study, heritability estimates for T1D and RA were comparatively small at $19.00 \%$ and $10.00 \%$, respectively. However, $h^{2}$ estimates vary widely in literature; T1D estimates range between 40 and $92 \%{ }^{3}$ while RA estimates range between 12 and $60 \%{ }^{11-13}$. Estimates also depend on the underlying data or the method used in the analysis. For example, twin studies result in broad sense heritability estimates where epigenetics are not taken into account. This increases the risk of inflated estimates ${ }^{3,50}$. Furthermore, sample size and pedigree information affect the ability to estimate genetic parameters and thus the power to detect significance. The depth of the pedigrees was theoretically sufficient to derive imprinting variances, but coancestry information between the maternal and paternal gametes of individuals with RA phenotypes would be a requirement for imprinting variance components analyses. In this context, the availability of genealogy databases in combination with genotypic data would increase the traceability of coancestries. Examples of such databases include the Utah Population database and a number of reliable Icelandic database $s^{51,52}$. The availability of population-scale family trees ${ }^{53}$ would further allow the determination of the parental origin of alleles ${ }^{54}$ and generally enable large scale human population studies on epidemiological history ${ }^{55}$. Overall, the reliability of data and its impact on T1D and RA diagnoses must be discussed. According to Ludvigsson et al. (2011) the ratio of correct diagnoses for RA in the Swedish Hospital Discharge Register is $93.5 \%$ and $87.1 \%$ with and without lymphoma, respectively. In this study, no distinction was made between T1D and T2D from ICD-7 through ICD-9. T1D was therefore defined according the age at first hospitalisation as being not older than 20 years. For T1D and T2D 79\% of cases were correctly diagnosed. The Swedish Hospital Discharge Register has provided complete national coverage since 1987 with more than $99 \%$ of all somatic hospital discharges currently registered ${ }^{56}$.

The replicability of the results of this study depends on the available data basis. Our results are based on data collected until 2007 (RA) and 2012 (T1D). The constant upgrade of the Swedish Hospital Discharge Register, supported by an increasing digitization of data collections, will further improve the size of good quality data and increase the pedigree depth so that higher power to detect significance can be expected when fitting our methods. Furthermore, while disease incidences in the data may not reflect the population incidences, the extension of data will lead to an accumulation of information on family affiliations as well as on the occurrence of the disorders across generations. This will improve the efficiency of estimating heritability in general and the separation of variance components in particular.

With regard to the impact of the mother, we found highly significant maternally derived environmental effects on T1D susceptibility. This finding is supported by Hirschhorn (2003) who stated that there are convincing data that non-genetic factors, such as environmental factors in early childhood, play a role in T1D susceptibility, while Nisticò et al. (2012) found significant effects of the shared environment on T1D susceptibility in an Italian twin cohort study ${ }^{57}$. Maternal environmental effects are expected to especially affect an individual in utero, perinatally, or during early childhood (familial environment) ${ }^{58}$. Factors such as early exposure to cow milk and cereals or a shortened duration of breastfeeding have been mentioned in this context ${ }^{58}$. In addition, we found the mother's SEI to have a significant effect on T1D susceptibility in her offspring. It should however be considered that the SEI 
of a mother is in itself influenced by her environment. Examples include her husband's SEI or other life circumstances. Apart from indications for the importance of maternal environmental effects, corresponding variance component analyses in full-sib populations could indicate the existence of an autosomal dominant inheritance pattern. However, based on findings from studies in Finland and the University of Southern California, Jerram and Leslie (2017) concluded that T1D susceptibility is unlikely to be affected by autosomal dominant genes ${ }^{59}$.

With regard to RA, the maternal genetic variance was found to be significant when maternal environmental effects were not part of the model. Moreover, results indicated an inflation of both, the additive genetic and the maternal environmental variance by maternal genetic effects if ignored in the models. The threshold model results underline these findings. Maternal genetic effects on RA susceptibility have not been reported before. However, as sex-linked effects such as X-chromosomal and mitochondrial effects were not considered in the analysis, an inflation of the maternal genetic variance cannot be excluded. The role of the X-chromosome for the development of RA has previously been discussed; however, these studies have focused on the impact of skewed X-chromosomal inactivation in the context of sex differences in RA susceptibility ${ }^{60,61}$. In addition, the existence of maternal environmental effects on RA susceptibility cannot be excluded. In utero effects, which include maternal smoking ${ }^{62}$ or protective effects of maternal non-inherited HLA-antigens ${ }^{63}$, breast-feeding in perinatal life $e^{64}$, or hygiene standards during postnatal development ${ }^{44}$ have been reported. This study found that RA development was affected by the mother's SEI. To conclude, while their relative importance could not clearly be quantified, both maternal genetic and environmental effects are indicated in RA susceptibility. Furthermore, while the ability to estimate covariances depends on the population structure ${ }^{65}$, the existence of covariances between additive and maternal genetic effects is nevertheless possible given that the $h^{2}$ value was reduced to zero when a maternal genetic effect was added to the various models.

Regardless, our study clearly indicated the significance of maternal effects on the development of T1D and RA. This was important as some of the contributory factors could be modified, possibly leading to the prevention of disease or treatment interventions ${ }^{66}$.

Environmental and sex effects. The development of T1D and RA were found to be significantly different over the last century when considering the effects of the year of birth. With regard to T1D, Gale (2002) stated that an increase in the incidence thereof over the second half of the twentieth century within a genetically relatively stable population would imply that environmental factors play a role in its etiology ${ }^{67}$. Hence, birth year effects could be attributed to environmental factors which have changed during the last century. There is a reason to suspect that these factors are linked to adjustments in living conditions which are, among others, affected by the economic state of a country. The Swedish economy has been characterised by a steady acceleration in economic growth with decelerations observed during the 1970 s and early $1980 \mathrm{~s}^{68}$. Increased disposable incomes are usually associated with improved living conditions. That living conditions can have an effect on T1D susceptibility is supported by our finding that the SEI of the mother, and thus the environment she provides for her children, significantly affects their likelihood of being diagnosed with T1D. In earlier studies, the susceptibility to T1D has also been shown to be associated with increased, lifestyle-associated linear growth and obesity ${ }^{69}$. While T1D susceptibility varied between regions, medical and the presumed corresponding residential regions were found to significantly affect the likelihood of being diagnosed with this condition. This finding is supported by Tzaneva et al. (2001), who reported that the onset of T1D is strongly dependent on the area of residence ${ }^{70}$. Nevertheless, as observed in Fig. 3, the varying effects associated with the medical regions and year of birth might also be due to the periodic and regional variation in the construction of the Swedish Hospital Discharge Register and Outpatient Register. While the Swedish Hospital Discharge Register was founded in 1964 in six Swedish counties mainly located in the Uppsala region, its nationwide launch was only in 1986. Notably, the Swedish Hospital Discharge Register is now almost 100\% with lower coverage of hospital-based outpatient care (approximately $80 \%)^{56}$.

As rheumatoid arthritis usually occurs later in life, it cannot accurately be determined which environmental factors have operated during a lifetime. There is evidence that an immune system can be permanently modified by environmental factors at an early age, with growth, nutrition and infectious exposure already having activated the immune system before disease onset ${ }^{44}$. This was supported by our novel finding that the SEI of the mother significantly affected RA susceptibility in children and that the SEI of the individual affected RA susceptibility later in life. The latter effect is also influenced by environmental factors such as the intake of oral contraceptives ${ }^{71}$. In addition, an inverse relationship between the total number of children and RA susceptibility was found. Pregnancy has been reported to typically ameliorate symptoms of $\mathrm{RA}^{72}$ and breast feeding is known to decrease RA susceptibility ${ }^{64}$. With regard to men, causal effects remain to be discussed. It should be noted that while the effect of the number of children on RA susceptibility was not investigated separately in women and men, significant interactions between sex and the number of children underline existing sex-associated differences. The difficulty in determining when environmental triggers occurred in individual life times may be reflected in the challenging endeavor of estimating the effects of medical regions on disease susceptibility. No information was available for how long individuals have resided in the analysed regions, potentially explaining the small effects and large standard errors. However, as for T1D, the periodic and regional variation in the data collection might also be a reason for this ${ }^{56}$. Nevertheless, geographical variation in RA incidence has previously been reported. Indications were found for the lower incidence rates in South European countries compared to North American and north European countries ${ }^{73,74}$.

In the context of sex differences in T1D and RA susceptibility, effects of the X-chromosome, sex hormones, sex-specific behavior, and sex-specific differences in body composition and structure have been discussed ${ }^{32,60,61,75,76}$. Our study confirmed that there was a significant sex effect in the development of both disorders. Moreover, especially for T1D, significant interactions between sex and birth year were found. This 
raises the question whether environmental effects preferentially interact with either sex. Based on the hypothesis that the prevalence of T1D amongst males increases proportionally in relation to disease incidence when the underlying environmental causes preferentially affect males, Gale and Gillespie (2001) reviewed sex ratios in T1D-incidence in multiple populations. They found that, although disease incidence increased, the sex ratio does not change and the trend towards male cases is specific for some populations ${ }^{75}$. They concluded that environmental effects do not interact with males over females. In our study, the effect of sex on T1D was only visible from 1960 to the mid-1980s.

Linear versus threshold model. One difficulty in this study was that the affection status was measured as a categorical trait with binomial distributions. Therefore, the linear models are not appropriate to analyze this data type. Nevertheless, statistical significance of genetic parameters could only be tested using the REML log-likelihood of linear models via RLRT. When a threshold model is used, the ASReml-package employs an approximate likelihood (penalised quasi-likelihood) that cannot be used to test differences ${ }^{77}$. There are currently no alternatives to the ASReml-package for our specific imprinting analysis as it is the only package that allows setting an appropriate correlation between the two parental gametic effects. This function ensures equivalence between the Mendelian and imprinting models. Equivalence is needed to perform an RLRT. Regarding the utilisation of linear and threshold models, both models generated similar results in uncovering the underlying genetic variation for T1D and RA. High correlations between the estimated genetic values indicated a fairly good fit of both models to the data.

\section{Conclusion}

Not only was new knowledge gained on the environmental effects on T1D and RA development, the separate contributions of each POE was able to delineate the genetic and phenotypic variation in T1D and RA susceptibility for the first time. Results supported findings that imprinting was of minor importance, but confirmed the role maternal factors played in the occurrence of both diseases. The prospects of fitting complex genetic variance-covariance structures can be expected to further improve given the size of good quality epidemiological data and pedigree depth.

\section{Material}

General data. This study was based on Swedish population-based registries with national coverage. Registry entries were linked using each person's unique identification number. To ensure participant confidentiality, this identification number was replaced by a serial number. The project registry was linked to the Multigeneration Register and Population Registers providing information on family relations, and SEI (in Swedish, socioekonomisk indelning), medical region and birth year, respectively. Individuals diagnosed with AIs were identified from the Swedish Hospital Discharge Register (contains data regarding hospitalisation and diagnoses in some regions since 1964 and nationwide since $1986^{78}$ ) and from the Outpatient Register since 2001. For further information see Hemminki (2001) and Hemminki et al. (2009) $)^{79,80}$.

Type 1 diabetes. The data selection for T1D was strict. T1D was not defined as an independent entity until the ICD-10 (International Classification of Diseases) classification system ${ }^{80}$. Therefore, T1D was defined through first hospitalisation until an age of 20 years in addition to the ICD codes (ICD-7 code 250, ICD-8 code 260 , ICD-9 code 260 or ICD-10 code E10) to unambiguously delineate T1D from type 2 diabetes ${ }^{81}$. Furthermore, as AIs were recorded from 1964, it was assumed that the health status of individuals born prior to 1944 cannot be unambiguously defined. Therefore, only individuals born after 1943 were assigned a case/control status (Fig. 5). Furthermore, only individuals born in Sweden and with known maternal data were considered in the analyses. Overall 27,255 T1D patients (14,626 male and 12,629 female) and 43,856 controls (20,234 male and 23,622 female) were used for the analyses. All individuals were born between 1944 and 2012. The age at diagnosis ranged between zero and 20 years with a mean age of 11 years $(s d=5.16$; Supplementary Fig. S4). Ancestral data was extracted from the Multigeneration Register for all patients. The complete dataset included 208,114 individuals (103,434 male and 104,680 female), with birth years ranging between 1862 and 2012. The dataset contained five generations with 111,626 founders and 23,124 families. The smallest family contained two individuals, and the largest 91 individuals. The average number of cases per family was 1.18 (the lowest number was one case per family; the highest number was 12 cases per family).

Rheumatoid arthritis. To not erroneously assign an individual with RA to the control status, health status was only declared for individuals born after 1938, i.e. for individuals not older than 25 years in 1964 when AIs registration was initiated (Fig. 5). Cases were selected according to the following codes: ICD-7 code 722, ICD-8 codes 712.0, 712.1, and 712.3, ICD-9 code 714, and ICD-10 codes M05, M06, M08.0, and M08.2. Only individuals born in Sweden and with known maternal data were considered in the analyses. Overall 15,850 patients (4,408 male and 11,442 female) diagnosed with RA and 5,199 controls (2,358 male and 2,841 female) were used for the analyses. They were born between 1939 and 2007. The age at diagnosis ranged between zero and 73 years with a mean age of 46.29 years ( $s d=16.65$; Supplementary Fig. S4). Ancestral data was extracted from the Multigeneration Register for all patients. The generated dataset included 60,684 individuals (26,339 male and 34,345 female), with birth years ranging between 1875 and 2007. The dataset contained five generations with 37,142 founders and 15,314 families. The smallest family contained two individuals, and the largest 21 individuals. The average number of cases per family was 1.04 (the lowest number was one case per family; the highest number was six cases per family). 

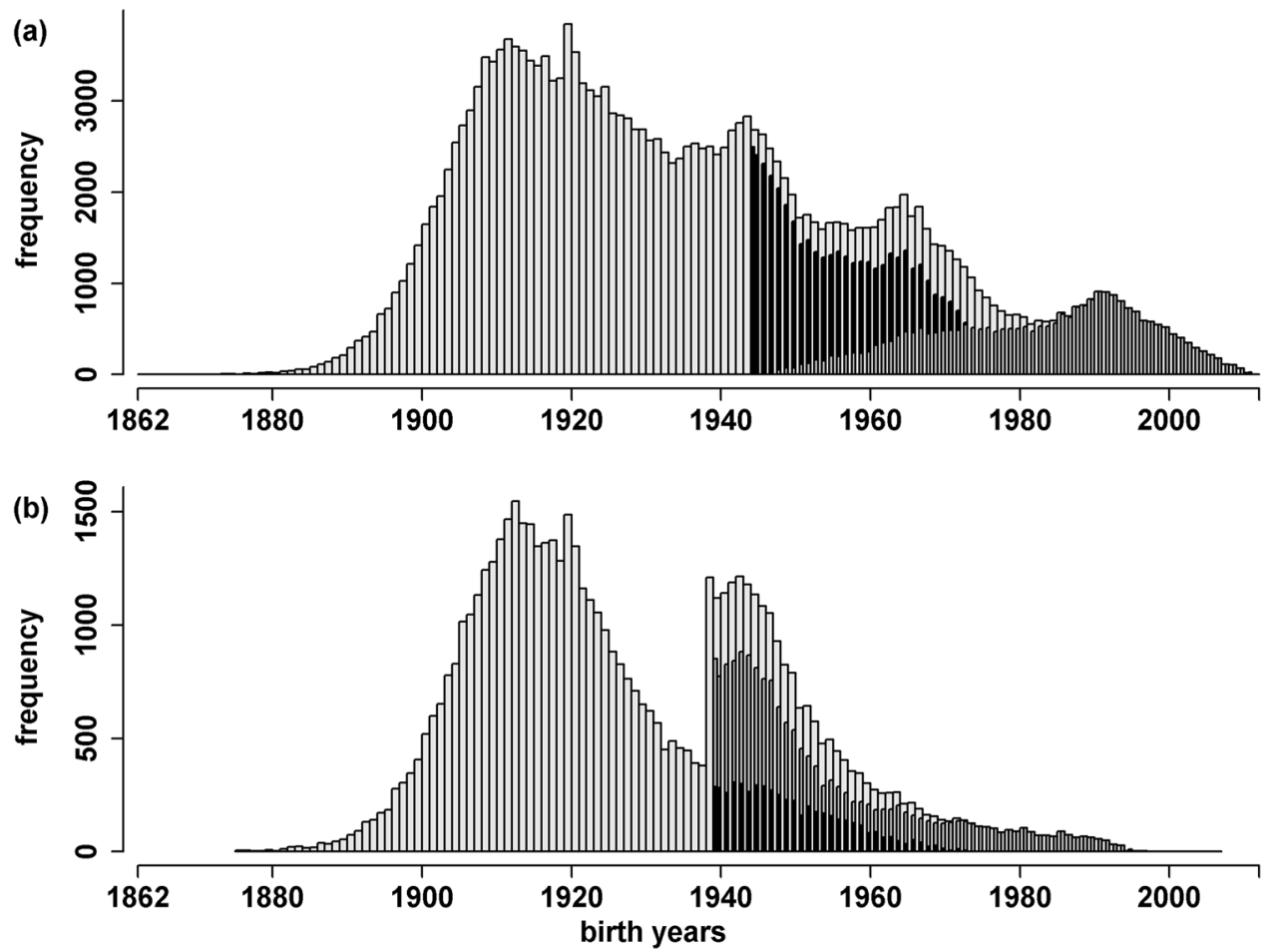

Figure 5. Distribution of birth years of all individuals in the pedigrees (light gray) as well as of all controls (black) and cases (gray) of type 1 diabetes mellitus (a) and rheumatoid arthritis (b).

\section{Methods}

The Mendelian models. To test the significance of the imprinting variance, a nested, equivalent version of the imprinting model must be applied. This model predicts a reduced number of parameters. It is called the Mendelian model as it represents the null-hypothesis that imprinting does not exist, i.e. the two parental gametes are not expressed differently (pure Mendelian inheritance). It can be written as:

$$
\boldsymbol{Y}=\boldsymbol{X} \boldsymbol{b}+\boldsymbol{Z}_{\boldsymbol{g}} \boldsymbol{g}+\boldsymbol{e}, \quad \text { (Mendelian model 1) }
$$

where $Y$ is a vector of the response variable; $\boldsymbol{b}$ is a vector of fixed effects; $\boldsymbol{g}$ is the vector of random gametic effects; $\boldsymbol{X}$ and $Z_{g}$ are incidence matrices; and $\boldsymbol{e}$ is the vector of random residuals. They are assumed to be normally distributed with a mean of 0 and variances $G \sigma_{g}^{2}$.

Model extensions. Other POEs such as maternal genetic or maternal environmental effects have been reported to be potential nuisance factors, i.e. erroneously assumed to be maternal effects due to imprinting, if not considered within the model definitions ${ }^{41}$. To be able to distinguish maternal effects due to imprinting from other POEs as well as to investigate the importance of the latter, the following versions of Mendelian model 1 were applied:

$$
\begin{aligned}
& Y=X b+Z_{g} g+Z_{c} c+e, \\
& Y=X b+Z_{g} g+Z_{c} c+Z_{m} m+e,
\end{aligned}
$$

(Mendelian model 2)

(Mendelian model 3)

where $\boldsymbol{c}$ and $\boldsymbol{m}$ are vectors of random maternal environmental and maternal genetic effects, respectively. They are assumed to be normally distributed with a mean of 0 and variances $\boldsymbol{I}_{\boldsymbol{c}} \sigma_{c}^{2}$ and $\boldsymbol{A} \sigma_{m}^{2}$. Matrix $\boldsymbol{I}_{\boldsymbol{c}}$ is an identity matrix and $\boldsymbol{A}$ is the additive genetic relationship matrix; the incidence matrices $\boldsymbol{Z}_{c}$, and $\boldsymbol{Z}_{m}$ relate observations and random effects. The significance of the additional random maternal environmental effect in Mendelian model 2 was tested by comparing the REML log-likelihood values of Mendelian model 1 and Mendelian model 2 using a one-sided RLRT. The RLRT is assumed to be $\chi^{2}$-distributed with a degree of freedom (DF) of one. The significance of the maternal genetic effect in Mendelian model 3 was tested by comparing the REML log-likelihood values of Mendelian model 2 and Mendelian model 3 using a one-sided RLRT $\left(\chi^{2}\right.$-distributed with DF $\left.=1\right)$.

As described for T1D, the maternal environmental variance turned out to be significant. Therefore, the following imprinting model was used to investigate the significance of the imprinting variance in T1D:

$$
Y=X b+Z_{s} g_{s}+Z_{d} g_{d}+Z_{c} c+e .
$$

This model is equivalent to Mendelian model 2 but assumes different contributions of maternal and paternal gametes to the susceptibility to T1D. 
With regard to RA, the existence of maternal environmental and maternal genetic effects could not be excluded. Hence, the imprinting model was applied with an additional maternal environmental effect (see above) but also with an additional maternal genetic effect:

$$
Y=X b+Z_{s} g_{s}+Z_{d} g_{d}+Z_{m} m+e .
$$

In general, the significance of the imprinting variance was determined by comparing the REML log-likelihood value of the imprinting model with the REML log-likelihood outcome of the corresponding Mendelian models using a one-sided RLRT. The test statistic was assumed to be asymptotically distributed as a mixture of two $\chi^{2}$ distributions with $\mathrm{DF}=1$ and $\mathrm{DF}=2^{36-40,82}$.

Calculation of population parameters. For the Mendelian models the direct heritabilities were calculated as $h_{\text {Mendel }}^{2}=\sigma_{a}^{2} / \sigma_{p}^{2}$, where $\sigma_{a}^{2}=2 \sigma_{g}^{2}$ and $\sigma_{p}^{2}=\sigma_{g}^{2}+\sigma_{e}^{2}$ in Mendelian model $1, \sigma_{p}^{2}=\sigma_{g}^{2}+\sigma_{e}^{2}+\sigma_{c}^{2}$ in Mendelian model 2, and $\sigma_{p}^{2}=\sigma_{g}^{2}+\sigma_{e}^{2}+\sigma_{c}^{2}+\sigma_{m}^{2}$ in Mendelian model 3. The latter two expressions of $\sigma_{p}^{2}$ were used to calculate $c_{\text {Mendel }}^{2}\left(c_{\text {Mendel }}^{2}=\sigma_{c}^{2} / \sigma_{p}^{2}\right)$ for Mendelian model 2 and Mendelian model 3, respectively. To calculate $m_{\text {Mendel }}^{2}\left(m_{\text {Mendel }}^{2}=\sigma_{m}^{2} / \sigma_{p}^{2}\right)$ for Mendelian model 3, $\sigma_{p}^{2}$ was defined as $\sigma_{p}^{2}=\sigma_{g}^{2}+\sigma_{e}^{2}+\sigma_{c}^{2}+\sigma_{m}^{2}$.

For the imprinting models the direct heritabilities were calculated as $h_{i m p}^{2}=\sigma_{a}^{2} / \sigma_{p}^{2}$, where $\sigma_{a}^{2}=\sigma_{g s}^{2}+\sigma_{g d}^{2}$ and $\sigma_{p}^{2}=\sigma_{g s}^{2}+\sigma_{g d}^{2}+\sigma_{e}^{2}+\sigma_{c}^{2}$ or $\sigma_{p}^{2}=\sigma_{g s}^{2}+\sigma_{g d}^{2}+\sigma_{e}^{2}+\sigma_{m}^{2}$. The first expression of $\sigma_{p}^{2}$ can be used to calculate $c_{i m p}^{2}$ as $c_{i m p}^{2}=\sigma_{c}^{2} / \sigma_{p}^{2}$. The latter expression of $\sigma_{p}^{2}$ can be used to calculate $m_{i m p}^{2}\left(m_{i m p}^{2}=\sigma_{m}^{2} / \sigma_{p}^{2}\right)$.

Threshold model. As the case/control-status is denoted $1 / 0$, the trait can be assumed binomially distributed. Hence, apart from the linear mixed models, which were needed for hypotheses testing, generalised linear mixed models (threshold models) were applied using a logit link and the pseudo-likelihood approach of Gilmour et al. $(2015)^{77}$. The probability that an observation with index $k$ belongs to class zero is:

$$
\pi\left(\eta_{k}\right)=\exp \left(\eta_{k}\right) /\left[1+\exp \left(\eta_{k}\right)\right]
$$

where - using Mendelian model 2 as an example - the linear predictor is:

$$
\eta_{k}=x_{k} \beta+z_{g, k} g+z_{c, k} c
$$

and $x_{k}, z_{g, k}$, and $z_{c, k}$ are the $k$ th rows of the aforementioned incidence matrices $\boldsymbol{X}, \boldsymbol{Z}_{\boldsymbol{g}}$, and $\boldsymbol{Z}_{\boldsymbol{c}}$ and the vectors $\boldsymbol{\beta}, \boldsymbol{g}$ and $c$ are defined as described for Mendelian model 2.

Fixed effects. Fixed effects included sex ( 2 levels; $1=$ male, $2=$ female), birth year ( 68 birth years for T1D; 57 birth years for RA), medical region (26 levels; individuals lived in 25 medical regions [26= unknown]), and the SEI of the mother ( 6 levels) for which the following codes were used:

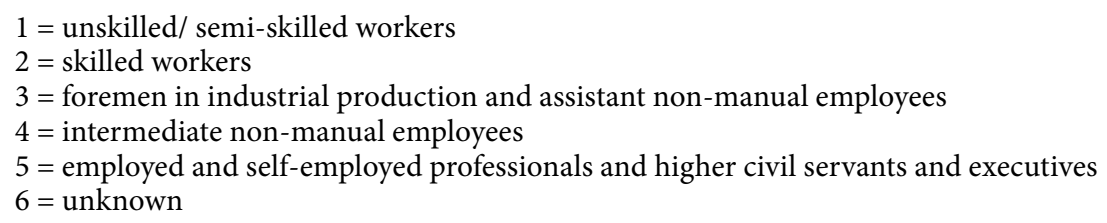

Note that coding differed across the various versions of the population database. Therefore, they were adjusted and equalised according to the 2012 version. The time an individual was under observation was considered by including the Legendre polynomials from the years under observation up to order three (linear, quadratic and cubic). Additional effects included in the RA model were the SEI of the individual (6 levels), whether an individual was a single child or had any siblings ( 2 levels; $1=$ single child, $2=$ siblings), and the number of offspring (12 levels; range of $0-11$ children). Furthermore, interactions between sex and birth years were fitted for both disorders. Interactions between sex and SEIs, as well as sex and the number of offspring were considered for RA.

Note that due to the underrepresentation of individuals in birth year levels, individuals were shifted to the next birth year level so that at least five individuals were obtained for each level.

The significance of all fixed effects was tested using an incremental Wald $F$ test implemented within the statistical software package ASReml version $4.2^{77}$. Note that ASReml caters for linear dependencies in the model by setting singular effects to zero ${ }^{83}$. Variance-covariance components were also estimated via ASReml. The R-packages "kinship2" version $1.6 .4^{84}$ and "pedigree" version $1.4^{85}$ in R version 3.5.2 $2^{86}$ were used to prepare the data.

Coordinates of Sweden used for Fig. 3 and Supplementary Fig. S2 were downloaded from https:/www.scb.se/ hitta-statistik/regional-statistik-och-kartor/regionala-indelningar/digitala-granser/ (accessed in November 2019) in the ArcView-shape format. Data were edited using the "readOGR" function implemented in the R-package "rgdal" version $1.4-8^{87}$ which was used in R version 3.6.1

\section{Data availability}

Individual-based data for research purposes is protected by strict confidentiality protections imposed by the Swedish Government and applied by Statistics Sweden, but can be made available for research after an ethical 
review and a review by both Statistics Sweden and the Swedish National Board of Health and Welfare. The Swedish National Board of Health and Welfare may be contacted for data access using the following link: https://www. socialstyrelsen.se/statistik-och-data/statistik/.

Received: 11 March 2020; Accepted: 18 June 2020

Published online: 14 July 2020

\section{References}

1. Wang, L., Wang, F. S. \& Gershwin, M. E. Human autoimmune diseases: a comprehensive update. J. Intern. Med. 278, 369-395 (2015).

2. Mathis, D., Vence, L. \& Benoist, C. B-cell death during progression to diabetes. Nature 414, 792-798 (2001).

3. Pociot, F. Type 1 diabetes genome-wide association studies: not to be lost in translation. Clin. Transl. Immunol. 6, e162 (2017).

4. Calabresi, E., Petrelli, F., Bonifacio, A. F., Puxeddu, I. \& Alunno, A. One year in review 2018: pathogenesis of rheumatoid arthritis. Clin. Exp. Rheumatol. 36, 175-184 (2018).

5. Silman, A. J. \& Pearson, J. E. Epidemiology and genetics of rheumatoid arthritis. Arthritis Res. 4, 265-272 (2010).

6. Stankov, K., Benc, D. \& Draskovic, D. Genetic and epigenetic factors in etiology of diabetes mellitus type 1. Pediatrics 132, $1112-1122(2013)$.

7. Hirschhorn, J. N. Genetic epidemiology of type 1 diabetes. Pediatr. Diabetes 4, 87-100 (2003).

8. Cho, J. H. \& Gregersen, P. K. Genomics and the multifactorial nature of human autoimmune disease. N. Engl. J. Med. 365, $1612-1623$ (2011).

9. Kim, K., Bang, S. Y., Lee, H.-S. \& Bae, S.-C. Update on the genetic architecture of rheumatoid arthritis. Nat. Rev. Rheumatol. 13, 13-24 (2016).

10. Deane, K. D. et al. Genetic and environmental risk factors for rheumatoid arthritis. Best Pract. Res. Clin. Rheumatol. 31, 3-18 (2017).

11. MacGregor, A. J. et al. Characterizing the quantitative genetic contribution to rheumatoid arthritis using data from twins. Arthritis Rheum. 43, 30-37 (2000).

12. Frisell, T. et al. Familial risks and heritability of rheumatoid arthritis: role of rheumatoid factor/anti-citrullinated protein antibody status, number and type of affected relatives, sex, and age. Arthritis Rheum. 65, 2773-2782 (2013).

13. Svendsen, A. J. et al. On the origin of rheumatoid arthritis: the impact of environment and genes-a population based twin study. PLoS ONE 8, e57304 (2013).

14. Xiang, Z., Yang, Y., Chang, C. \& Lu, Q. The epigenetic mechanism for discordance of autoimmunity in monozygotic twins. J. Autoimmun. 83, 43-50 (2017).

15. Generali, E., Ceribelli, A., Stazi, M. A. \& Selmi, C. Lessons learned from twins in autoimmune and chronic inflammatory diseases. J. Autoimmun. 83, 51-61 (2017).

16. Jerram, S. T., Dang, M. N. \& Leslie, R. D. The role of epigenetics in type 1 diabetes. Curr. Diab. Rep. 17, 89 (2017).

17. Blunk, I. \& Reinsch, N. in Proceedings of the 10th World Congress on Genetics Applied to Livestock Production (2014).

18. Yu, S. et al. Variable and tissue-specific hormone resistance in heterotrimeric Gs protein alpha-subunit (Gsalpha) knockout mice is due to tissue-specific imprinting of the gsalpha gene. Proc. Natl. Acad. Sci. U. S. A. 95, 8715-8720 (1998).

19. Gould, T. D. \& Pfeifer, K. Imprinting of mouse Kvlqt1 is developmentally regulated. Hum. Mol. Genet. 7, 483-487 (1998).

20. Lawson, H. A., Cheverud, J. M. \& Wolf, J. B. Genomic imprinting and parent-of-origin effects on complex traits. Nat. Rev. Genet. 14, 609-617 (2013).

21. Ferguson-Smith, A. C. Genomic imprinting: the emergence of an epigenetic paradigm. Nat. Rev. Genet. 12, 565-575 (2011).

22. Morison, I. M., Ramsay, J. P. \& Spencer, H. G. A census of mammalian imprinting. Trends Genet. 21, 457-465 (2005).

23. Plasschaert, R. N. \& Bartolomei, M. S. Genomic imprinting in development, growth, behavior and stem cells. Development 141, 1805-1813 (2014).

24. Angulo, M. A., Butler, M. G. \& Cataletto, M. E. Prader-Willi syndrome: a review of clinical, genetic, and endocrine findings. J. Endocrinol. Invest. 38, 1249-1263 (2015).

25. Margolis, S. S., Sell, G. L., Zbinden, M. A. \& Bird, L. M. Angelman syndrome. Neurotherapeutics 12, 641-650 (2015).

26. Enfield, K. S. et al. Deregulation of small non-coding RNAs at the DLK1-DIO3 imprinted locus predicts lung cancer patient outcome. Oncotarget 7, 80957-80966 (2016).

27. Denic, S. \& Agarwal, M. M. Breast cancer protection by genomic imprinting in close kin families. BMC Med. Genet. 18, 136 (2017).

28. Warram, J. H., Krolewski, A. S., Gottlieb, M. S. \& Kahn, C. R. Differences in risk of insulin-dependent diabetes in offspring of diabetic mothers and diabetic fathers. N. Engl. J. Med. 311, 149-152 (1984).

29. McCarthy, B. J., Dorman, J. S. \& Aston, C. E. Investigating genomic imprinting and susceptibility to insulin-dependent diabetes mellitus: an epidemiologic approach. Genet. Epidemiol. 8, 177-186 (1991).

30. Guo, S. W. \& Tuomilehto, J. Preferential transmission of type 1 diabetes from parents to offspring: fact or artifact?. Genet. Epidemiol. 23, 323-334 (2002).

31. Wallace, C. et al. The imprinted DLK1-MEG3 gene region on chromosome $14 \mathrm{q} 32.2$ alters susceptibility to type 1 diabetes. Nat. Genet. 42, 68-71 (2010).

32. Van Vollenhoven, R. F. Sex differences in rheumatoid arthritis: more than meets the eye. BMC Med. 7, 12 (2009).

33. Martin-Trujillo, A. et al. Loss of imprinting of IGF2 characterises high IGF2 mRNA-expressing type of fibroblast-like synoviocytes in rheumatoid arthritis. Ann. Rheum. Dis. 69, 1239-1242 (2010).

34. Zhou, X. et al. Joint linkage and imprinting analyses of GAW 15 rheumatoid arthritis and gene expression data. BMC Proc. 1(Suppl 1), S53 (2007).

35. Mitchell, B. D. \& Pollin, T. I. Genomic imprinting in diabetes. Genome Med. 2, 55 (2010).

36. Neugebauer, N., Luther, H. \& Reinsch, N. Parent-of-origin effects cause genetic variation in pig performance traits. Animal 4, 672-681 (2010).

37. Neugebauer, N., Rader, I., Schild, H. J., Zimmer, D. \& Reinsch, N. Evidence for parent-of-origin effects on genetic variability of beef traits. J. Anim. Sci. 88, 523-532 (2010).

38. Blunk, I., Mayer, M., Hamann, H. \& Reinsch, N. Parsimonious model for analyzing parent-of-origin effects related to beef traits in dual-purpose Simmental. J. Anim. Sci. 95, 559-571 (2017).

39. Blunk, I., Mayer, M., Hamann, H. \& Reinsch, N. A parsimonious model for an analysis of parent-of-origin effects on beef traits in dual-purpose Simmental. Adv. Anim. Biosci. 8, s76-s78 (2017).

40. Blunk, I., Mayer, M., Hamann, H. \& Reinsch, N. A new model for parent-of-origin effect analyses applied to Brown Swiss cattle slaughterhouse data. Animal 11, 1096-1106 (2017).

41. Hager, R., Cheverud, J. M. \& Wolf, J. B. Maternal effects as the cause of parent-of-origin effects that mimic genomic imprinting. Genetics 178, 1755-1762 (2008).

42. Falconer, D. S. Introduction to Quantitative Genetics (Oliver \& Boyd, Great Britain, 1960).

43. Willham, R. L. Problems in estimating maternal effects. Livest. Prod. Sci. 7, 405-418 (1980). 
44. Edwards, C. J. \& Cooper, C. Early environmental factors and rheumatoid arthritis. Clin. Exp. Immunol. 143, 1-5 (2006).

45. Blunk, I., Mayer, M., Hamann, H. \& Reinsch, N. Scanning the genomes of parents for imprinted loci acting in their un-genotyped progeny. Sci. Rep. 9, 654 (2019).

46. Engellandt, T. \& Tier, B. Genetic variances due to imprinted genes in cattle. J. Anim. Breed. Genet. 119, 154-165 (2002).

47. Schaeffer, L. R., Kennedy, B. W. \& Gibson, J. P. The inverse of the gametic relationship matrix. J. Dairy Sci. 72, 1266-1272 (1989).

48. Gibson, J. P., Kennedy, B. W., Schaeffer, L. R. \& Southwood, O. I. Gametic models for estimation of autosomally inherited genetic effects that are expressed only when received from either a male or female parent. J. Dairy Sci. 71, 143 (1988).

49. Bartok, B. \& Firestein, G. S. Fibroblast-like synoviocytes: key effector cells in rheumatoid arthritis. Immunol. Rev. 233, 233-255 (2010).

50. Mayhew, A. J. \& Meyre, D. Assessing the heritability of complex traits in humans: methodological challenges and opportunities. Curr. Genom. 18, 332-340 (2017).

51. Stefansdottir, V. et al. The use of genealogy databases for risk assessment in genetic health service: a systematic review. J. Community Genet. 4, 1-7 (2012).

52. Cannon Albright, L. A. Utah family-based analysis: past, present and future. Hum. Hered. 65, 209-220 (2008).

53. Kaplanis, J. et al. Quantitative analysis of population-scale family trees with millions of relatives. Science 360, 171-175 (2018).

54. Kong, A. et al. Parental origin of sequence variants associated with complex diseases. Nature 462, 868-874 (2009).

55. Shor, T., Kalka, I., Geiger, D., Erlich, Y. \& Weissbrod, O. Estimating variance components in population scale family trees. PLoS Genet. 15, e1008124 (2019).

56. Ludvigsson, J. F. et al. External review and validation of the Swedish national inpatient register. BMC Public Health 11, 450 (2011).

57. Nisticò, L. et al. Emerging effects of early environmental factors over genetic background for type 1 diabetes susceptibility: evidence from a Nationwide Italian Twin Study. J. Clin. Endocrinol. Metab. 97, E1483-E1491 (2012).

58. Rewers, M. \& Ludvigsson, J. Environmental risk factors for type 1 diabetes. Lancet 387, 2340-2348 (2016).

59. Jerram, S. T. \& Leslie, R. D. The genetic architecture of type 1 diabetes. Genes (Basel) 8, E209 (2017).

60. Chabchoub, G. et al. Analysis of skewed X-chromosome inactivation in females with rheumatoid arthritis and autoimmune thyroid diseases. Arthritis Res. Ther. 11, R106 (2009).

61. Kanaan, S. B. et al. Evaluation of X chromosome inactivation with respect to HLA genetic susceptibility in rheumatoid arthritis and systemic sclerosis. PLoS ONE 11, e0158550 (2016).

62. Jaakkola, J. J. K. \& Gissler, M. Maternal smoking in pregnancy as a determinant of rheumatoid arthritis and other inflammatory polyarthropathies during the first 7 years of life. Int. J. Epidemiol. 34, 664-671 (2005).

63. Feitsma, A. L. et al. Protective effect of noninherited maternal HLA-DR antigens on rheumatoid arthritis development. Proc. Natl. Acad. Sci. U. S. A. 104, 19966-19970 (2007).

64. Karlson, E. W., Mandl, L. A., Hankinson, S. E. \& Grodstein, F. Do breast-feeding and other reproductive factors influence future risk of rheumatoid arthritis? Results from the Nurses' Health Study. Arthritis Rheum. 50, 3458-3467 (2004).

65. Heydarpour, M., Schaeffer, L. R. \& Yazdi, M. H. Influence of population structure on estimates of direct and maternal parameters. J. Anim. Breed. Genet. 125, 89-99 (2008).

66. Paschou, S. A., Papadopoulou-Marketou, N., Chrousos, G. P. \& Kanaka-Gantenbein, C. On type 1 diabetes mellitus pathogenesis. Endocr. Connect. 7, R38-R46 (2018).

67. Gale, E. A. The rise of childhood type 1 diabetes in the 20th century. Diabetes 51, 3353-3361 (2002).

68. Schön, L. Sweden-economic growth and structural change, 1800-2000. EH.Net Encyclopedia, https://eh.net/encyclopedia/swede n-economic-growth-and-structural-change-1800-2000/ (2008).

69. Hyppönen, E., Virtanen, S. M., Kenward, M. G., Knip, M. Åkerblom, H. K., \& Childhood Diabetes in Finland Study Group. Obesity, increased linear growth, and risk of type 1 diabetes in children. Diabetes Care 23, 1755-1760 (2000).

70. Tzaneva, V., Iotova, V. \& Yotov, Y. Significant urban/rural differences in the incidence of type 1 (insulin-dependent) diabetes mellitus among Bulgarian children (1982-1998). Pediatr. Diabetes 2, 103-108 (2001).

71. Doran, M. F., Crowson, C. S., O'Fallon, W. M. \& Gabriel, S. E. The effect of oral contraceptives and estrogen replacement therapy on the risk of rheumatoid arthritis: a population based study. J. Rheumatol. 31, 207-213 (2004).

72. Williams, W. V. Hormonal contraception and the development of autoimmunity: a review of the literature. Linacre Q. 84, 275-295 (2017).

73. Costenbader, K. H., Chang, S.-C., Laden, F., Puett, R. \& Karlson, E. W. Geographic variation in rheumatoid arthritis incidence among women in the United States. Arch. Intern. Med. 168, 1664-1670 (2008).

74. Alamanos, Y., Voulgari, P. V. \& Drosos, A. A. Incidence and prevalence of rheumatoid arthritis, based on the 1987 American College of Rheumatology criteria: a systematic review. Semin. Arthritis Rheum. 36, 182-188 (2006).

75. Gale, E. A. \& Gillespie, K. M. Diabetes and gender. Diabetologia 44, 3-15 (2001).

76. Kovacs, W. J. \& Olsen, N. J. Sexual dimorphism of RA manifestations: genes, hormones and behavior. Nat. Rev. Rheumatol. 7, 307-310 (2011).

77. Gilmour, A. R., Gogel, B. J., Cullis, B. R., Welham, S. J. \& Thompson, R. ASReml User Guide Release 4.2 Functional Specification (VSN International Ltd, Hemel Hempstead). (2015).

78. Hemminki, K., Bevier, M., Sundquist, J. \& Hemminki, A. Cancer of unknown primary (CUP): does cause of death and family history implicate hidden phenotypically changed primaries?. Ann. Oncol. 23, 2720-2724 (2012).

79. Hemminki, K. Genetic epidemiology_science and ethics on familial cancers. Acta Oncol. 40, 439-444 (2001).

80. Hemminki, K., Li, X., Sundquist, J. \& Sundquist, K. Familial association between type 1 diabetes and other autoimmune and related diseases. Diabetologia 52, 1820-1828 (2009).

81. Landgren, O. et al. Patterns of autoimmunity and subsequent chronic lymphocytic leukemia in nordic countries. Blood 108, 292-296 (2006).

82. Self, S. G. \& Liang, K. Y. Asymptotic properties of maximum likelihood estimators and likelihood ratio tests under nonstandard conditions. J. Am. Stat. Assoc. 82, 605-610 (1987).

83. Gilmour, A. R. ASREML for testing fixed effects and estimating multiple trait variance components. Proc. Adv. Anim. Breed. Gen. 12, 386-390 (1997).

84. Sinnwell, J. P., Therneau, T. M. \& Schaid, D. J. The kinship2 R package for pedigree data. Hum. hered. 78, 91-93 (2014).

85. Coster, A. Pedigree: pedigree functions. R package version 1.4 (2013).

86. R Core Team. R: A Language and Environment for Statistical Computing (R Foundation for Statistical Computing, Vienna, 2018).

87. Bivand, R., Keitt, T. \& Rowlingson, B. rgdal: Bindings for the 'Geospatial' Data Abstraction Library. R package version 1.4-8. (2019).

88. R Core Team. R: A Language and Environment for Statistical Computing (R Foundation for Statistical Computing, Vienna, 2019).

\section{Acknowledgements}

The project was funded by the Deutsche Forschungsgemeinschaft (DFG, German Research Foundation) - 418890112, by the Swedish Research Council to Jan Sundquist (2016-01176) and to Kristina Sundquist (2018-02400) as well as by the Swedish Heart-Lung Foundation (20180465) and ALF funding (in Swedish, 
Avtal om Läkarutbildning och Forskning) Region Skåne awarded to Kristina Sundquist. The authors gratefully acknowledge support by A. Eggert, B. Garske, S. Chattopadhyay, O. Bandapalli, and M.-Å. Persson.

\section{Author contributions}

I.B. created the design of the study, carried out the analyses, interpreted the results, and prepared the manuscript. H.T. assisted the data retrieval, supported the analyses, and participated in the design of the study and preparation of the manuscript. N.R. and M.M. supported the analyses, especially on statistical matters, and participated in the interpretation of data and preparation of the manuscript. A.F., J.S., K.S., and K.H. supported the analyses and assisted in the preparation of the manuscript. All authors revised it critically for important intellectual content and approved the final manuscript.

\section{Competing interests}

The authors declare no competing interests.

\section{Additional information}

Supplementary information is available for this paper at https://doi.org/10.1038/s41598-020-68212-x.

Correspondence and requests for materials should be addressed to I.B.

Reprints and permissions information is available at www.nature.com/reprints.

Publisher's note Springer Nature remains neutral with regard to jurisdictional claims in published maps and institutional affiliations.

(c) (i) Open Access This article is licensed under a Creative Commons Attribution 4.0 International License, which permits use, sharing, adaptation, distribution and reproduction in any medium or format, as long as you give appropriate credit to the original author(s) and the source, provide a link to the Creative Commons license, and indicate if changes were made. The images or other third party material in this article are included in the article's Creative Commons license, unless indicated otherwise in a credit line to the material. If material is not included in the article's Creative Commons license and your intended use is not permitted by statutory regulation or exceeds the permitted use, you will need to obtain permission directly from the copyright holder. To view a copy of this license, visit http://creativecommons.org/licenses/by/4.0/.

(C) The Author(s) 2020 